\title{
Nitric Oxide and Hydrogen Peroxide Production are Involved in Systemic Drought Tolerance Induced by 2R,3R-Butanediol in Arabidopsis thaliana
}

\author{
Song-Mi Cho ${ }^{1 \dagger}$, Yong Hwan Kim ${ }^{2 \dagger}$, Anne J. Anderson ${ }^{3}$ and Young Cheol Kim ${ }^{4 *}$ \\ ${ }^{1}$ Department of Floriculture, Chunnam Techno University, Jeonnam 516-911, Korea \\ ${ }^{2}$ Korea Institute of Planning \& Evaluation for Technology on Food, Agriculture, Forestry \& Fisheries, Anyang 431-060, Korea \\ ${ }^{3}$ Department of Biology, Utah State University, Logan, UT 84322-5305, USA \\ ${ }^{4}$ Institute of Environmental-Friendly Agriculture, College of Agriculture and Life Sciences, Chonnam National University, Gwangiu \\ 500-757, Korea \\ (Received on July 17, 2013; Revised on August 30, 2013; Accepted on August 31, 2013)
}

\begin{abstract}
2R,3R-Butanediol, a volatile compound produced by certain rhizobacteria, is involved in induced drought tolerance in Arabidopsis thaliana through mechanisms involving stomatal closure. In this study, we examined the involvement of nitric oxide and hydrogen peroxide in induced drought tolerance, because these are signaling agents in drought stress responses mediated by abscisic acid (ABA). Fluorescence-based assays showed that systemic nitric oxide and hydrogen peroxide production was induced by $2 \mathrm{R}, 3 \mathrm{R}$-butanediol and correlated with expression of genes encoding nitrate reductase and nitric oxide synthase. Co-treatment of 2R,3Rbutanediol with an inhibitor of nitrate reductase or an inhibitor of nitric oxide synthase lowered nitric oxide production and lessened induced drought tolerance. Increases in hydrogen peroxide were negated by cotreatment of 2R,3R-butanediol with inhibitors of NADPH oxidase, or peroxidase. These findings support the volatile 2R,3R-butanediol synthesized by certain rhizobacteria is an active player in induction of drought tolerance through mechanisms involving nitric oxide and hydrogen peroxide production.
\end{abstract}

Keywords : abscisic acid, induced drought tolerance, nitric oxide, reactive oxygen, volatiles

Signal transduction pathways are involved in the perception of water deficiency in plants. The plant hormone, abscisic acid (ABA) is a major chemical signal. Drought stress triggers the production of $\mathrm{ABA}$ to stimulate responses involved in drought tolerance in plants (Shinozaki and Yamaguchi-Schinozaki, 2000). Downstream physiological changes include stomatal closure, decreased photosynthesis,

\footnotetext{
These authors contributed equally to this study

* Corresponding author.

Phone) +82-62-530-2071, FAX) +82-62-530-2079

E-mail)yckimyc@jnu.ac.kr
}

and accumulation of compatible solutes (Lee and Luan, 2012; Shinozaki and Yamaguchi-Schinozaki, 2000). ABA accumulates in the roots of drought-stressed plants and moves upward to the leaves, where stomatal closure is induced. Consequently, ABA biosynthetic mutants ( $a b a$ mutants) and some ABA-response mutants (abi mutants) impaired in the regulation of stomatal aperture are very susceptible to water stress (Leung and Giraudat, 1998; Schroeder et al., 2001).

ABA signaling is interlinked with other pathways involving nitric oxide (NO), ethylene, salicylic acid (SA), and jasmonic acid (JA) with consequences that are antagonistic or synergistic (Anderson et al., 2004; Desikan et al., 2006; Leung and Giraudat, 1998; Mosher et al., 2010). Single exogenous applications of ABA, ethylene, methyl jasmonic acid, or NO induce stomatal closure whereas scavenging NO prevents stomatal closure (Neill et al., 2008). However, ethylene disrupts stomatal closure when added with ABA or NO (Desikan et al., 2006; Neil et al., 2008). ABA generally negatively regulates SA, JA, and ethylene-dependent pathways for pathogen resistance (Anderson et al., 2004; Mosher et al., 2010). The reactive oxygen species, hydrogen peroxide, also functions in ABA-mediated drought tolerance (Lee and Luan, 2012). Thus, pathway interactions involved in regulating stomatal closure are complex.

Root colonization by certain microbes induces drought tolerance (Kim et al., 2012). Induction of drought tolerance is correlated with colonization by the Gram-positive bacteria, Paenibacillus polymyxa (Figueiredo et al., 2008; Timmusk and Wagner, 1999) and a Bacillus subtilis strain (Yang et al., 2010; Zhang et al., 2010), as well as an endophytic fungus and a Trichoderma species (Bae et al., 2009). Gramnegative bacterial isolates, an ACC-deaminase producing bacterium (Mayak et al., 2004), and Pseudomonas chlororaphis O6 (Cho et al., 2008, 2011) also induce systemic tolerance to pathogens.

The mechanisms involved in microbe-mediated drought 
tolerance are not well understood. Both $B$. subtilis and $P$. chlororaphis $\mathrm{O} 6$ produce the volatile, 2R,3R-butanediol (Cho et al., 2008; Han et al., 2006; Ryu et al., 2003). This product promotes plant growth, and induces systemic resistance against bacterial pathogens, and drought tolerance (Cho et al., 2008, 2011; Han et al., 2006; Ryu et al., 2003). For example, partial stomatal closure and an enhanced rate of closure upon drought stress accompany colonization of Arabidopsis roots by P. chlororaphis O6 (Cho et al., 2012). Our recent transcriptome analysis of Arabidopsis thaliana reveals that several plant defense related transcripts were primed by root colonization of $P$. chororaphis $\mathrm{O} 6$ and could be a key element of induced systemic drought tolerance by O6 strain (Cho et al., 2013).

In this study, we examined whether NO and hydrogen peroxide played a role in drought tolerance induced by $P$. chlororaphis O6 root colonization. We assayed for production of $\mathrm{NO}$ and hydrogen peroxide in plants treated with 2R,3R-butanediol using fluorescent indicators. We also used a characterized scavenger of $\mathrm{NO}$ and inhibitors of enzymes involved in NO- and hydrogen peroxide-synthesis to observe their effects on the responses triggered by 2R,3R-butanediol. The effects of 2R,3R-butanediol on expression of genes encoding the two nitrate reductases (NRs) and NO synthase (NOS) in Arabidopsis were compared to elucidate differences in the pathways. Our results indicate that 2R,3R-butanediol is a volatile microbial associated molecular pattern (MAMP)-like factor causing priming events that function in the tolerance of plants to both abiotic and abiotic stresses, including drought resistance.

\section{Materials and Methods}

Plant material and growth conditions. Arabidopsis seeds (Columbia ecotype) were surface-sterilized and maintained at $4{ }^{\circ} \mathrm{C}$ for vernalization. A single germinated seed was placed on the surface of sterile Whatman no. 1 filter paper placed over agar, 0.3\% Phytagel, containing half-strength Murashige Skoog (MS) salt medium (Murashige and Skoog, 1962) modified with 3\% sucrose at pH 5.7. This medium was contained in the wells of sterile 12-well plates (SPL Lifescience Co., Pecheon, Korea). Plates were sealed with Parafilm to prevent drying, and no additional water was supplied during the experiment. Seedlings were grown with a cycle of $16 \mathrm{~h}$ light $/ 8 \mathrm{~h}$ dark under $40-\mathrm{W}$ fluorescent lights $\left(2,000 \mathrm{lux}, 80 \mu \mathrm{mol}\right.$ photon $\left.\mathrm{m}^{-2} \mathrm{~s}^{-1}\right)$. The temperature was maintained at $22 \pm 1{ }^{\circ} \mathrm{C}$ with relative humidity of $50-$ $60 \%$.

Measurement of induced systemic drought tolerance. The roots of 2-week-old well-plate grown seedlings were treated with $10 \mu \mathrm{l}$ of $100 \mu \mathrm{M}$ 2R,3R-butanediol (Sigma
Aldrich Inc., St. Louis, MO, USA) in the presence and absence of $0.2 \mathrm{mM} \mathrm{N \omega}$-nitro-L-arginine (L-NNA, SigmaAldrich), an inhibitor of nitric oxide synthase, or $0.2 \mathrm{mM}$ 2-phenyl-4,4,5,5-tetramethylimidazoline-1-oxyl-3-oxide (PTIO), a NO scavenger. As a control, sterile MES buffer (25 mM MES-KOH [pH 6.15] containing $10 \mathrm{mM} \mathrm{KCl),}$ L-NNA and PTIO was applied to the roots. Three days after root treatment, the plants on the filter paper were transferred to open, empty Petri dishes. The extent of plant wilting was assessed visually after $2 \mathrm{~h}$, and the number of the plants that survived after adding water was counted. This experiment was performed three times with 20 plants per treatment.

Gene expression analysis by reverse transcription polymerase chain reaction (RT-PCR). Ten two week-old seedlings were transferred to a new 6-well plate and roots were treated with $3 \mathrm{~mL}$ of $100 \mu \mathrm{M}$ 2R,3R-butanediol or $100 \mu \mathrm{M}$ ABA. Total RNAs from the upper portion (shoot and leaves) of the chemical treated plants were isolated using an RNeasy Plant Mini Kit (Qiagen Inc., Valencia, CA, USA) at $0,12,24,48$, and $72 \mathrm{~h}$ after treatments. Realtime quantification was conducted in a Rotor-Gene 2000 real-time thermal cycling system (Corbett Research, Sydney, Australia) using a QuantiTect SYBR Green RT-PCR kit (Qiagen). The reaction mixture $(25 \mu \mathrm{L})$ contained $500 \mathrm{ng}$ of total RNA, $0.5 \mu \mathrm{M}$ of each primer, and appropriate amounts of enzymes and fluorescent dyes, according to the manufacturer's instructions (Qiagen). The control where the cDNA was omitted produced no PCR products.

Expression from the Arabidopsis actin 1 gene was used as an internal standard. The primers were: forward: 5'-CAT CAG GAA GGA CTT GTA CGG-3', reverse: 5'-GAT GGA CCT GAC TCG TCA TAC-3'. Primers for the genes involved in NO synthesis were: AtNR1 (forward: 5'-CTC AGT TCA TGG TGC CTC AA-3', reverse: 5'-CAC GGG TAA ACC AAG CTG TT-3'); AtNR2 (forward: 5'-CTT TGG TAG ACG CCG AAC TC-3', reverse: 5'-CTC CAC ACG GGT CAC TTT TT-3'); AtNOS1 (forward: 5'-CAG CGG CAC AAA AGT ACA AA-3', reverse: 5'-ATC GAA AGA TTG GCC TCT GA-3'). The data from the PCR runs were analyzed using the software provided by the manufacturer. Three plants/treatment were used for each transcript analysis, and data represent means of three independent experiments.

In vivo detection of hydrogen peroxide in plants. Hydrogen peroxide was detected visually in the leaves of plants using 3,3'-diaminobenzidine (DAB) as a substrate for endogenous peroxidase activity. Briefly, three week-old intact plants raised in the well plates were treated with $2 \mathrm{R}, 3 \mathrm{R}$ butanediol $(10,100,1,000 \mu \mathrm{M})$, SA $(2 \mathrm{mM})$, or sterile 
water (buffer) as a control, by spraying the leaves. After 6 $\mathrm{h}$, individual plant leaves were excised at the base of the petiole, transferred to 12 -well plates, and immersed in 1 $\mathrm{mg} / \mathrm{mL}$ DAB (pH 3.8) for $8 \mathrm{~h}$ under light at $25^{\circ} \mathrm{C}$. The leaves were decolorized by immersion in $96 \%$ methanol for $10 \mathrm{~min}$. The presence of hydrogen peroxide was indicated by the deep brown pigmentation from the reaction between DAB and hydrogen peroxide catalyzed by plant peroxidases. The leaves were observed under a stereoscopic microscope (JP/PM20, Olympus, Tokyo, Japan) and images were acquired using Motic image plus 2.0 (Motic China Group Co., Ltd, City, China) software. Each treatment was performed with three separate plants, and the entire experiment was repeated twice.

Detection of NO production in guard cells. Leaf epidermal peels of three weeks-old Arabidopsis plants, were incubated for $3 \mathrm{hr}$ in MES buffer ( $25 \mathrm{mM}$ MES-KOH, $\mathrm{pH}$ 6.15 , and $10 \mathrm{mM} \mathrm{KCl}$ ), were transferred to $15 \mu \mathrm{M} \mathrm{4}$, 5diaminofluorescein diacetate (DFDA) (Sigma-Aldrich) diluted in MES buffer for $20 \mathrm{~min}$, and washed three times in MES buffer as described in the protocol used by Melloto et al. (2006). The epidermal peel fragments were incubated for an additional $2 \mathrm{~h}$ in the presence of defined concentrations of chemicals that have an impact on NO formation using MES buffer as a control. All images were visualized using confocal laser scanning microscopy (excitation 488 $\mathrm{nm}$, emission $515 \mathrm{~nm}$; Olympus FV500). Images were analyzed using SCION IMAGE software (Scion Inc., Frederick, $\mathrm{MD}$, USA). Data are presented as mean pixel intensities, and 50-100 guard cells were observed per treatment of three independent replicates.

Detection of hydrogen peroxide production in guard cells. $\mathrm{H}_{2} \mathrm{O}_{2}$ production was monitored using the $\mathrm{H}_{2} \mathrm{O}_{2}$ sensitive fluorescent probes, 2,7-dichlorofluorescein-diacetate/ 2,7-dichlorofluorescein ( $\mathrm{H}_{2} \mathrm{DCFDA}$, Molecular Probes, Leiden, The Netherlands) using the methods described by Bright et al. (2006). Epidermal peels were loaded with $50 \mu \mathrm{M}$ $\mathrm{H}_{2}$ DCFDA in MES-KCl buffer for 15 min before washing in MES- $\mathrm{KCl}$ buffer for $20 \mathrm{~min}$. Hydrogen peroxide production was monitored after $2 \mathrm{~h}$ incubation with $2 \mathrm{R}, 3 \mathrm{R}$ butanediol in the absence or presence of diphenyleneiodonium (DPI, Molecular Probes) and salicylhdroxamic acid (SHAM, Molecular Probes) before the images were visualized. All images were visualized using confocal laser scanning microscopy (excitation $488 \mathrm{~nm}$, emission $515 \mathrm{~nm}$; Olympus FV500) and analyzed using SCION IMAGE software. Data are presented as mean pixel intensities, and 50-100 guard cells were observed per treatment for three independent replicates.

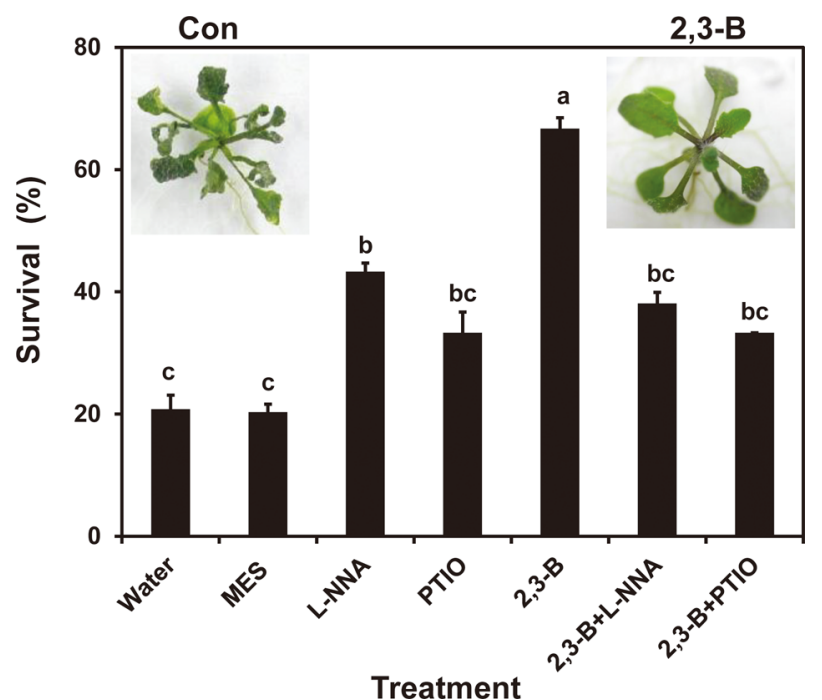

Fig. 1. Survival of Arabidopsis Col-0 plants through induced drought resistance generated by $2 \mathrm{R}, 3 \mathrm{R}$-butanediol $(2,3-\mathrm{B})$ in the presence and absence of chemicals to impair NO effects. Arabidopsis plants were grown in microtiter plates containing half-strength MS solid medium on sterile Whatman filter papers. Roots were treated with MES buffer as a control, or with $100 \mu \mathrm{M}$ 2R,3R-butanediol in MES buffer in the presence or absence of $0.2 \mathrm{mM} \mathrm{N} \omega$-nitro-L-arginine (L-NNA), and 0.2 mM 2-phenyl4,4,5,5-tetrmethyl-limidazolinone-1-oxyl-3-oxide (PTIO). The plants were exposed to drought stress after 3 days by transferring them to open Petri dishes, and wilted plants were counted after re-hydration. Three independent experiments were performed with at least 20 plants/treatment. Different letters indicate significant differences between treatments according to the least significant differences test at $P<0.05$. Representative photographic images of control (Con) or 2R,3R-butanediol-treated plants $(2,3-\mathrm{B})$ following drought stress are shown.

\section{Results}

Induction of drought tolerance by 2R,3R-butanediol. Survival after drought stress increased in seedlings where the roots were treated for 3 days with 2R,3R-butanediol from the $20 \%$ level for seedlings treated with water or MES buffer compared to $\geq 60 \%$ (Fig. 1). Treatments with an inhibitor of NO synthase, N $\omega$-nitro-L-arginine (L-NNA), or the NO scavenger, 2-phenyl-4,4,5,5-tetramethyl-limidazolinone-1-oxyl-3-oxide (PTIO), increased survival to above $35 \%$ (Fig. 1). When these chemicals were combined with 2R,3R-butanediol, plant survival was reduced to levels of the chemicals alone (Fig. 1). These findings were consistent with a role for $\mathrm{NO}$ in 2R,3R-butanediol-induced systemic drought tolerance.

Induction of nitric oxide by systemic application of 2R,3R-butanediol. Fluorescence generated from DAF2- 


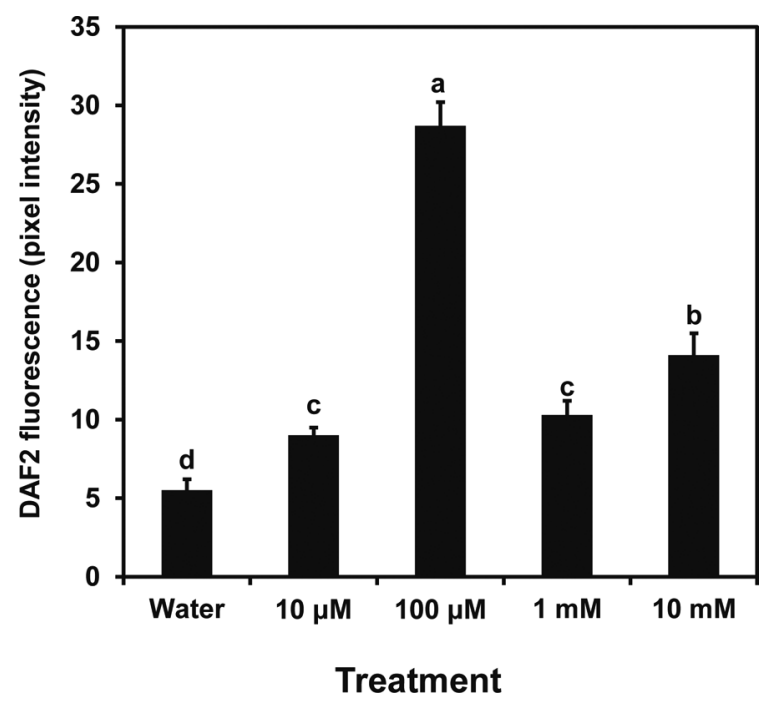

Fig. 2. Effect of different concentrations of 2R,3R-butanediol (2,3-B) treatment on $\mathrm{NO}$ generation. Leaf epidermal peel fragments were incubated with DAF2-DA in MES buffer. After 2 $\mathrm{h}$, the tissues were treated with 2R,3R-butanediol $(0,10 \mu \mathrm{M}, 100$ $\mu \mathrm{M}, 1 \mathrm{mM}$, and $10 \mathrm{mM})$ in MES buffer. Fluorescence was measured using a confocal microscope and data are displayed as mean pixel intensity and standard deviation. A total of 50-100 guard cells were observed per treatment of three independent replicates. Different letters indicate a significant difference between treatments according to the least significant differences test at $P<0.05$.

DA was used to determine the dose-response for induction of NO formation by butanediol. Figure 2 illustrates a maximum response with leaf treatments of $100 \mu \mathrm{M}$ butanediol with lower responses at higher and lower levels; $100 \mu \mathrm{M}$ 2R,3R-butanediol caused intense fluorescence in the guard cells (Fig. 3). This finding with the 2R,3Rbutanediol treatments is typical of hormesis observed in cell signaling systems, where there is a defined concentration for a maximal response and lesser response at higher and lower concentrations (Mattson 2008). No fluorescence was observed with treatments of MES buffer, PTIO, or L-NNA or when either L-NNA or PTIO was added with the 2R,3Rbutanediol. We explored the role of NOS or NR in NO generation further by using NG-nitro-L-arginine methyl ester (L-NAME), another inhibitor of NOS, and tungstate (Tug), an inhibitor of NR in the fluorescent assay. Both chemicals eliminated butanediol systemically-induced fluorescence (Fig. 4). These findings were consistent with butanediol inducing systemic NO production in the guard cells.

The finding of the potential roles of NOS and NR in the 2R,3R-butanediol response led us to examine expression from the genes encoding nitrate reductases and nitric oxide synthase. Findings were compared the responses to ABA as another inducer of drought resistance. The RT-PCR data revealed difference in timing of transcript formation at the
A
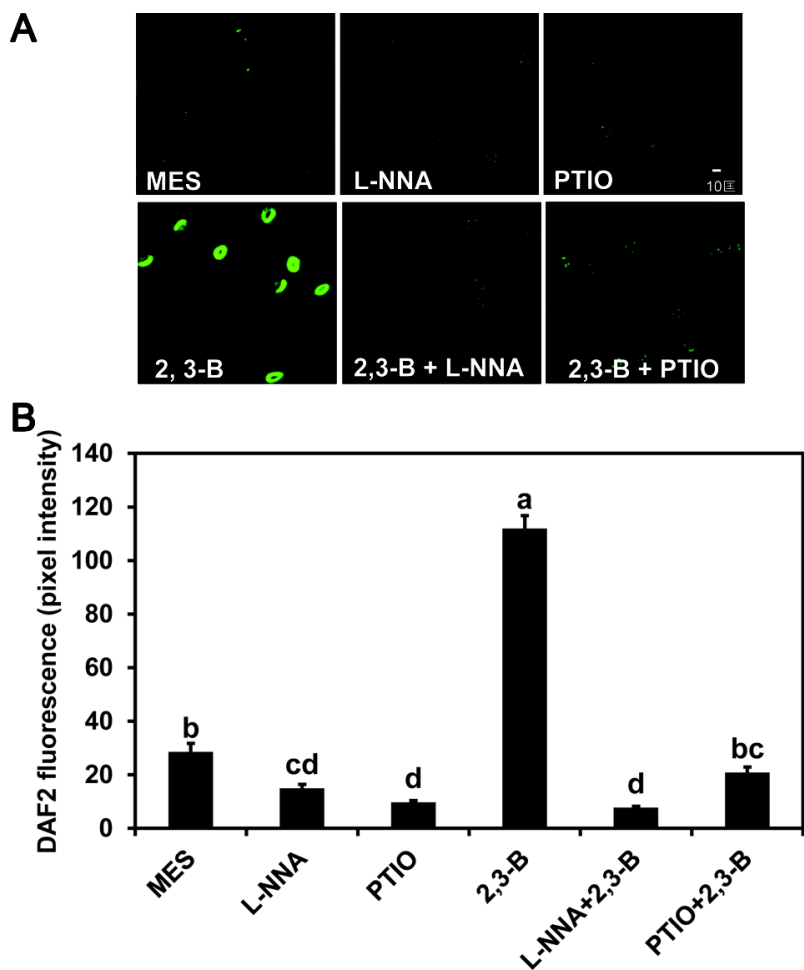

Fig. 3. Effect of NO inhibitors on induction of NO by $2 \mathrm{R}, 3 \mathrm{R}-$ butanediol (2,3-B). (A) DAF2-DA fluorescence in guard cells induced by $100 \mu \mathrm{M} 2 \mathrm{R}, 3 \mathrm{R}$-butanediol (2,3-B) was inhibited by the NO scavenger (PTIO) and an inhibitor of NO synthase (LNNA). Fluorescence of leaf peel epidermal fragments are shown after $2 \mathrm{~h}$ incubation with DAF2-DA in MES buffer following treatments with MES buffer as a control or $100 \mu \mathrm{M} 2 \mathrm{R}, 3 \mathrm{R}-$ butanediol $(2,3-\mathrm{B})$ in the presence or absence of L-NNA and PTIO. Fluorescence imaging due to the NO reaction with DAF2DA was observed by confocal microscopy with excitation at 488 $\mathrm{nm}$ and emission at $515 \mathrm{~nm}$. (B) Intensity of fluorescence is provided as mean pixel intensities and standard deviations based on observations of 50-100 hundred guard cells per treatment of three independent replications. Different letters indicate significant difference between treatments according to the least significant differences test at $P<0.05$.

dosages used for induction (Fig. 5). Both NR genes were induced with similar expression patterns by $2 \mathrm{R}, 3 \mathrm{R}$-butanediol with an early but transient transcript accumulation. Change in expression from the NOS gene was slower and to a lesser extent. Treatment with ABA induced AtNR1 to a greater extent than ATNR2 at $48 \mathrm{~h}$. Induction by ABA was to higher levels than with butanediol. Expression from At NOS1 at $24 \mathrm{~h}$ was considerably greater than for the $2 \mathrm{R}, 3 \mathrm{R}-$ butanediol treatment.

Induction of hydrogen peroxide by systemic application of 2R,3R-butanediol. Applying 2R,3R-butanediol to leaves generated hydrogen peroxide as evidenced by the formation of brown pigmentation in the presence of the peroxidase 


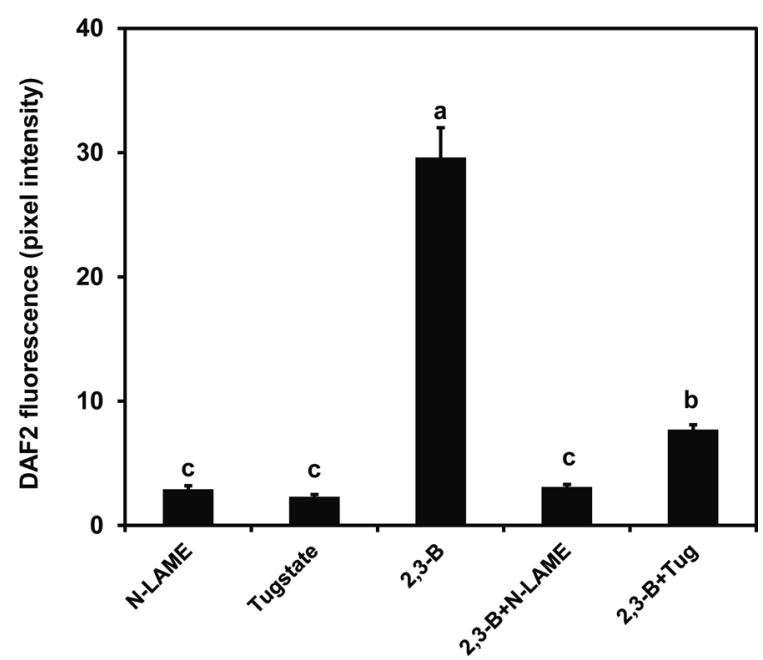

Fig. 4. Inhibition by tungstate (Tug) or NG-nitro-L-arginine methyl ester (L-NAME) of NO production stimulated by $2 \mathrm{R}, 3 \mathrm{R}$-butanediol (2,3-B). Epidermal cells in a leaf peel were incubated with DAF2-DA in MES buffer for $2 \mathrm{~h}$ prior to exposure to $100 \mu \mathrm{M}$ 2R,3R-butanediol (2,3-B) in the absence or presence of L-NAME $(200 \mu \mathrm{M})$ or tug $(100 \mu \mathrm{M})$. Fluorescence was measured using a confocal microscope, and data are displayed as mean pixel intensities and associated standard deviations. A total of 50-100 guard cells were observed per treatment of three independent replicates. Different letters indicate significant difference between treatments according to the least significant differences test at $P<0.05$.
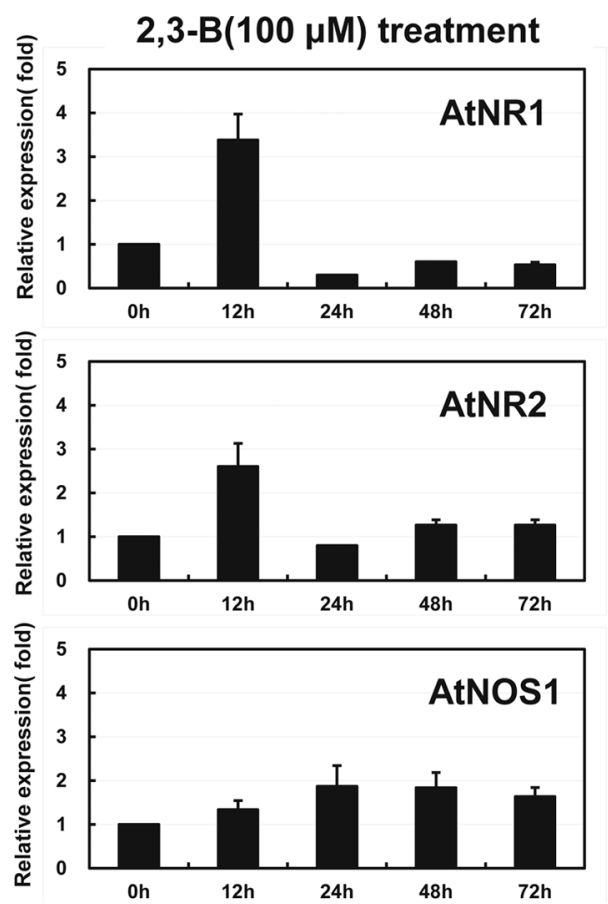

substrate, DAB (Fig. 6). This response was evident at 10 $\mu \mathrm{M}$ butanediol when the NO response was low (Fig. 2). We confirmed in Fig. 6 that hydrogen peroxide production by examination of leaves treated with SA (Mori et al., 2001). Formation of hydrogen peroxide was confirmed by observing the fluorescence in cells developing after $\mathrm{H}_{2}$ DCFDA loading (Fig 7). Fluorescence increased after 2R,3R-butanediol treatment (Fig. 7). The mechanism of hydrogen peroxide formation was assessed by additions of diphenyleneiodonium (DPI) to inhibit NADPH oxidase, or the peroxidase inhibitor salicylhydroxamic acid (SHAM). 2R,3R-butanediolinduced fluorescence was lowered by both chemicals, DPI and SHAM (Fig. 7).

\section{Discussion}

We have presented evidence that 2R,3R-butanediol, a microbially-produced inducer of drought tolerance in Arabidopsis, required pathways that generated systemically both hydrogen peroxide and NO synthesis. Chemicals that scavenged NO, or limited it synthesis, by inhibiting NR or NOS, prevented the 2R,3R-butanediol-stimulated NO accumulation in leaf epidermal cells and survival of intact seedlings under drought stress. Currently, we cannot explain the induc-
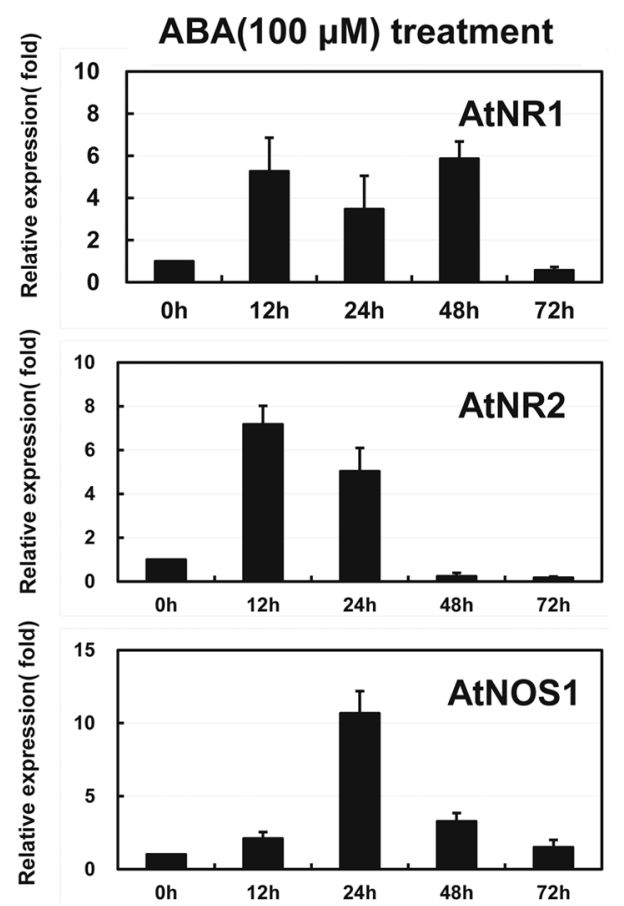

Fig. 5. Transcript accumulations from genes, AtNR1, AtNR2 and AtNOS1, encoding proteins involved in NO synthesis. Gene expression was determined with RNA extracted from plants without roots after seedlings were treated with $100 \mu \mathrm{M} 2 \mathrm{R}, 3 \mathrm{R}$-butanediol (2,3-B). Gene expression was analyzed by real-time RT-PCR in RNA extracted from Arabidopsis seedling that its roots treated with $100 \mu \mathrm{M} 2 \mathrm{R}, 3 \mathrm{R}-$ butanediol for $0,12,24,48$, and $72 \mathrm{~h}$. Specific primer sets were used to detect each gene expression pattern. Data were normalized based on actin gene expression. Vertical bars indicate \pm standard deviation of three independent experiments with three plants/treatment. Different letters indicate significant difference between treatments according to the least significant differences test at $P<0.05$. 

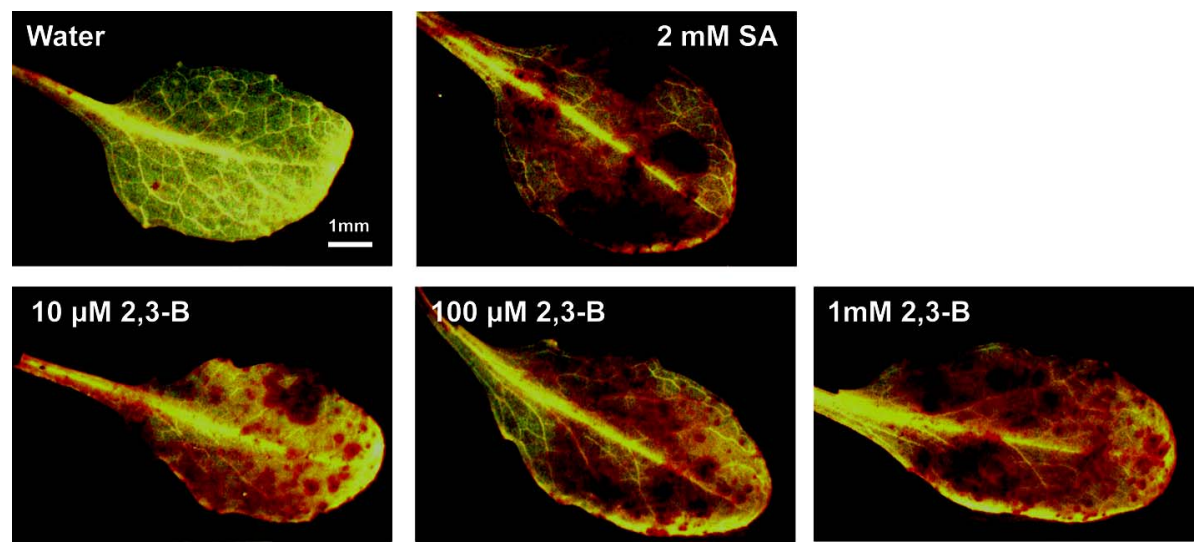

Fig. 6. 2R,3R-Butanediol (2,3-B) stimulated hydrogen peroxide formation in Arabidopsis leaves, a response duplicated by treatment with salicylic acid (SA). Three week-old plants were treated with water as a control, 2R,3R-butanediol $(10,100,1,000 \mu \mathrm{M})$ or $2 \mathrm{mM} \mathrm{SA}$ by spray application. Leaves were excised after $6 \mathrm{~h}$ and exposed to 3,3-diaminobenzidine (DAB) for $8 \mathrm{~h}$. The leaves were decolorized by immersion in $96 \%$ methanol for 10 min and photographed. The images are typical of three independent replicates with similar results.

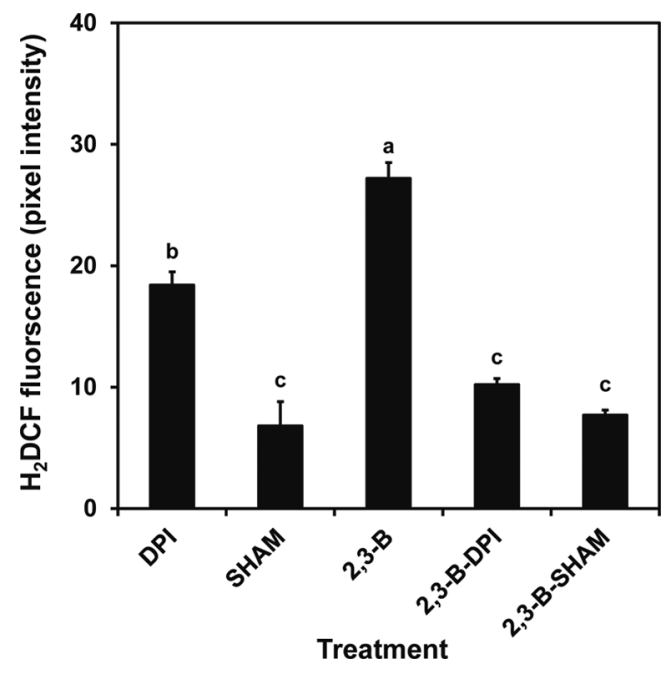

Fig. 7. Production of hydrogen peroxide stimulated by $2 \mathrm{R}, 3 \mathrm{R}-$ butanediol (2,3-B) is differentially sensitive to diphenyleneiodonium (DPI) or salicylhydroxamic acid (SHAM). Epidermal cells were incubated with $\mathrm{H}_{2}$ DCFDA in MES-KCl buffer as a control. The tissues were treated with MES-KCl buffer amended with $100 \mu \mathrm{M}$ 2R,3R-butanediol (2,3-B) in the absence or presence of DPI, an inhibitor of NADPH oxidase, and SHAM, an inhibitor of peroxidase. After two hours incubation, hydrogen peroxide production was monitored by assessing fluorescence using confocal microscopy with an excitation wavelength of $488 \mathrm{~nm}$ and an emission wavelength of $515 \mathrm{~nm}$. Fluorescence was measured using a confocal microscope and data are displayed as mean pixel intensity and associated standard deviations. A total of 50-100 guard cells were observed per treatment of three independent replicates. Different letters indicate significant difference between treatments according to the least significant differences test at $P<$ 0.05 .

tion of protection by the treatments with the chemicals LNNA or PTIO alone. We speculate that the response was related to the high complexity of the cell signaling mechanisms involving stomatal responses. A role for NR and NOS in the butanediol response was supported by the finding of increased transcript abundance from genes encoding NR and to a lesser extent NOS. 2R,3R-butanediol-induced hydrogen peroxide synthesis was revealed in intact leaves by allowing it to act as a substrate with DAB for plant peroxidases. The efficacy of the inhibitors of peroxidases (SHAM) and NADPH oxidase (DPI) indicated that both enzymes were needed to generate the response to butanediol.

Because 2R,3R-butanediol is produced by the root colonizer $P$. chlororaphis $\mathrm{O} 6$, and root colonization by this bacterium induces tolerance to drought (Cho et al., 2008, 2011), we propose that NO and hydrogen peroxide synthesis are part of the cell signaling system involved in these responses in the leaf tissue. Hydrogen peroxide production was observed in the 2R,3R-butanediol treatment of leaf epidermal cells. Thus, it appears that NADPH oxidase activity was essential for the 2R,3R-butanediol response. A previous study supported this concept; stomatal closure triggered by $P$. chlororaphis $\mathrm{O} 6$ was independent of ABA (Cho et al., 2012). The dose response relationship we observed in the induction of fluorescence indicative of NO formation with 2R,3R-butanediol is typical of hormesis, where there is an optimal dose for maximum responses. Thus our findings agree with studies in several systems, including reactive oxygen signaling, that also displayed the hormetic response (Mattson, 2008; Wetzer and Rubio, 2012).

The mechanism discussed for $P$. chlororaphis O6-induction of drought tolerance differs from that used by other root colonizers. For example the root colonizer Achromobacter piechaudii ARV8 reduced plant ethylene levels through the production of ACC deaminase (Mayak et al., 2004) which 
would reduce ethylene inhibition of $\mathrm{ABA}$-induced stomatal closure (Desikan et al., 2006; Neil et al., 2008).

Our findings demonstrated that microbial colonization induced both tolerance to abiotic stress and pathogen resistance. The production of $2 \mathrm{R}, 3 \mathrm{R}$-butanediol by $P$. chlororaphis O6 has been correlated with resistance to plant pathogens and tolerance to salt stress in other papers (Cho et al., 2008, 2010). Similar cross over protection occurs with B. subtilis GB03 root colonization (Zhang et al., 2010). Stomatal closure has been classified as part of innate immunity in plants to aid in disease resistance (Underwood et al., 2007). The flagellar peptide, flg22, and lipopolysaccharide (LPS) are bacterial MAMPs that induce stomatal closure when applied to leaves (Melotto et al., 2006). We also have demonstrated that flg22 and LPS induced drought tolerance (Cho et al., 2008). Disease resistance to pathogenic bacteria may be increased because the closure of the stomates restricted pathogen invasion (Kumar et al., 2012). However, Melotto et al., (2006) showed that pathogenic bacteria effectively override stomatal closure caused by MAMPs using other effectors, such as the toxin coronatine, to allow the interaction to proceed to a disease state. Such effectors are lacking in beneficial microbes.

MAMPs act to prime the induction of defense responses against microbial attack by decreasing the timing and increasing the intensity of defense responses when the plant is challenged by a pathogen (Melotto et al., 2006; Underwood et al., 2007). We postulated that the decrease in stomatal aperture incited by $P$. chlororaphis $\mathrm{O} 6$ root colonization also involved priming (Cho et al., 2010). Priming allowed the plant to respond more quickly with stomatal closure to subsequent drought stress. By these findings we have demonstrated that a volatile, the butanediol, acted as a priming agent in addition to conserved bacterial MAMP structures.

In summary, the volatile 2R,3R-butanediol, a product of rhizosphere-competent microbes, has been documented to act as an effector of induced drought tolerance. Volatility of the 2R,3R-butanediol would permit its activity in zones beyond the colonization site of the bacterium.

\section{Acknowledgements}

This study was supported by a grant from the Basic Science Research Program through the National Research Foundation of Korea (NRF) funded by the Ministry of Education, Science and Technology (2011-0011555).

\section{References}

Anderson, J. P., Badruzsufari, E., Schnek, P. M., Manners, J. M., Desmond, O. J., Ehlert, C., Maclean, D. J., Ebert, P. R. and
Kazan, K. 2004. Antagonistic interaction between abscisic acid and jasmonate-ethylene signaling pathways modulates defense gene expression and disease resistance in Arabidopsis. Plant Cell 16:3460-3479.

Bae, H., Sicher, R. C., Kim, M. S., Kim, S.-H., Strem, M. D., Melnick, R. L. and Bailey, B. A. 2009. The beneficial endophyte Trichoderma hamatum isolate DIS219b promotes growth and delays the onset of the drought response in Theobroma cacao. J. Exp. Bot. 60: 3279-3295.

Bright, J., Desikan, R., Hancock, J. T., Weir, I. S. and Neil, S. J. 2006. ABA-induced NO generation and stomatal closure in Arabidopsis are dependent on $\mathrm{H}_{2} \mathrm{O}_{2}$ synthesis. Plant J. 45: 113-122.

Cho, S. M., Kang, B. R., Han, S. H., Anderson, A. J., Park, J.-Y., Lee, Y.-H., Cho, B. H., Yang, K.-Y., Ryu, C.-M. and Kim, Y. C. 2008. 2R,3R-butanediol, a bacterial volatile produced by Pseudomonas chlororaphis O6, is involved in induction of systemic tolerance to drought in Arabidopsis thaliana. Mol. Plant-Microbe Interact. 21:1067-1075.

Cho, S. M., Kang, B. R., Kim, J. J. and Kim, Y. C. 2012. Induced systemic drought and salt tolerance by Pseudomonas chlororaphis $\mathrm{O} 6$ root colonization is mediated by $\mathrm{ABA}$-independent stomatal closure. Plant Pathol. J. 28:202-206.

Cho, S. M., Kang, B. R. and Kim, Y. C. 2013. Transcriptome analysis of induced systemic drought tolerance elicited by Pseudomonas chlororaphis O6 in Arabidopsis thaliana. Plant Pathol. J. 29:209-220.

Cho, S. M., Kim, S. H., Kim, Y. C., Yang, K. Y., Kim, K. S., Choi, Y. S. and Cho, B. H. 2010. Galactinol is involved in induced systemic resistance against bacterial infection and environmental stresses. Kor. J. Plant Res. 23:248-255.

Cho, S. M., Park, J. Y., Han, S. H., Anderson, A. J., Yang, K. Y., Gardener, B. M. and Kim, Y. C. 2011. Identification and transcriptional analysis of priming genes in Arabidopsis thaliana induced by root colonization with Pseudomonas chlororaphis O6. Plant Pathol. J. 27:272-279.

Desikan, R., Last, K., Harret-Williams, R., Tagliavia, C., Harter, K., Hooley, R., Hancock, J. T. and Neil, S. J. 2006. Ethyleneinduced stomatal closure in Arabidopsis occurs via atrbohFmediated hydrogen peroxide synthesis. Plant J. 47:907-917.

Figueiredo, M. V. B., Burity, H., Martinez, C. R. and Chanway, C. P. 2008. Alleviation of drought stress in the common bean (Phaseolus vulgaris L.) by co-inoculation with Paenibacillus polymyxa and Rhizobium tropici. Appl. Soil Eco. 40:182-188.

Han, S. H., Lee, S. J., Moon, J. H., Park, K. H., Yang, K. Y., Cho, B. H., Kim, K. Y., Kim, Y. H., Lee, M. C., Anderson, A. J. and Kim, Y. C. 2006. GacS-dependent production of 2R,3Rbutanediol by Pseudomonas chlororaphis $\mathrm{O} 6$ is a major determinant for eliciting systemic resistance against Erwinia carotovora but not against Pseudomonas syringae pv. tabaci in tobacco. Mol. Plant-Microbe Interact. 19:924-930.

Kim, Y. C., Glick, B. R., Bashan, Y. and Ryu, C.-M. 2012. Enhancement of plant drought tolerance by microbes. Aroca, R. (ed.), Plant responses to drought stress, Springer-Verlag, Berlin, Heidelberg, pp 383-413.

Kumar, A. S., Lakshmanan, V., Caplan, J. L., Powell, D., Czym- 
mek, K. J., Levia, D. F. and Bais, H. P. 2012. Rhizobacteria Bacillus subtilis restricts foliar pathogen entry through stomata. Plant J. 72:694-706.

Lee, S. C. and Luan, S. 2012. ABA signal transduction pathway at the crossroad of biotic and abiotic stress response. Plant Cell Environ. 35:53-60.

Leung, J. and Giraudat, J. 1998. Abscisic acid signal transduction. Annu. Rev. Plant Physiol. Plant Mol. Biol. 49:199-222.

Mayak, S., Tirosh, T. and Glick, B. R. 2004. Plant growth-promoting bacteria that confer resistance to water stress in tomatoes and peppers. Plant Sci. 166:525-530.

Mattson, M. P. 2008. Hormesis defined. Ageing Res Rev. 7:1-7.

Melloto, M., Underwood, W., Koczan, J., Nomura, K. and He, S. H. 2006. Plant stomata function in innate immunity against bacterial invasion. Cell 126:969-980.

Mori, I. C., Pinontoan, R., Kawano, T. and Muto, S. 2001. Involvement of superoxide generation in salicylic acidinduced stomatal closure in Vicia faba. Plant Cell Physiol. 42: 1383-1388.

Mosher, S., Moeder, W., Nishimura, N., Kikumaru, Y., Joo, S.-H., Urquhart, W., Klessig, D. F., Kim, S.-K., Nambara, E. and Yoshioka, K. 2010. The lesion-mimic mutant cpr22 shows alterations in abscisic acid signaling and abscisic acid insensitivity in a salicylic acid-depedent manner. Plant Physiol. 152:1901-1913.

Murashige, T. and Skoog, F. 1962. A revised medium for rapid growth and bioassay with tobacco tissue culture. Physiol. Plant. 15:473-497.

Neill, S., Barros, R., Bright, J., Desikan, R., Hancock, J., Harrision, J., Morris, P., Ribeiro, D. and Wilson, I. 2008. Nitric oxide, stomatal closure, and abiotic stress. J. Exp. Bot. 59: 165-176.

Ryu, C.-M., Farag, M. A., Hu, C.-H., Reddy, M. S., Wei, H.-X., Pare, P. W. and Kloepper, J. W. 2003. Bacterial volatiles promote growth in Arabidopsis. Proc. Natl. Acad. Soc. USA. 100: 4927-4932.

Schroeder, J. I., Kwak, J. M. and Allen, G. J. 2001. Guard cell abscisic acid signaling and engineering drought hardiness in plants. Nature 410:327-330.

Shinozaki, K. and Yamaguchi-Shinozaki, K. 2000. Molecular responses to dehydration and low temperature: differences and cross-talk between two stress signaling pathways. Curr. Opin. Plant Biol. 3:217-223.

Timmusk, S. and Wagner, E. G. H. 1999. The plant-growth-promoting rhizobacterium Paenibacillus polymyxa induces changes in Arabidopsis thaliana gene expression: a possible connection between biotic and abiotic stress responses. Mol. Plant-Microbe Interact. 12:951-959.

Underwood, W., Melloto, M. and He, S. H. 2007. Role of plant stomata in bacterial invasion. Cell Microbiol. 9:1621-1629.

Wetzker, R. and Rubio, I. 2012. Hormetic signaling patterns. Dose-Response 10:83-90.

Yang, J., Kloepper, J. W. and Ryu, C.-M. 2010. Rhizosphere bacteria help plants tolerance abiotic stress. Trends Plant Sci. 46: 1-3.

Zhang, H., Murzello, C., Sun, Y., Kim, M.-S., Xie, X., Jeter, R. M., Zak, J. C., Dowd, S. E. and Paré, P. W. 2010. Choline and osmotic-stress tolerance induced in Arabiodosis by the soil microbe Bacillus subtilis (GB03). Mol. Plant-Microbe Interact. 23:1097-1104. 